\title{
Calibration of the modulation transfer function of surface profilometers with binary pseudo-random test standards: Expanding the application range
}

\author{
Valeriy V. Yashchuk* ${ }^{\mathrm{a}}$, Erik H. Anderson ${ }^{\mathrm{a}}$, Samuel K. Barber ${ }^{\mathrm{a}}$, Nathalie Bouet ${ }^{\mathrm{b}}$, Rossana Cambie ${ }^{\mathrm{a}}$, \\ Raymond Conley ${ }^{\mathrm{b}}$, Wayne R. McKinney ${ }^{\mathrm{a}}$, Peter Z. Takacs ${ }^{\mathrm{c}}$, Dmitriy L. Voronov ${ }^{\mathrm{a}}$ \\ ${ }^{a}$ Lawrence Berkeley National Laboratory, Berkeley, California 94720 \\ ${ }^{\mathrm{b}}$ NSLS-II, Brookhaven National Laboratory Upton, New York 11973 \\ ${ }^{c}$ Brookhaven National Laboratory, Upton, New York 11973
}

\begin{abstract}
A modulation transfer function (MTF) calibration method based on binary pseudo-random (BPR) gratings and arrays [Proc. SPIE 7077-7 (2007), Opt. Eng. 47(7), 073602-1-5 (2008)] has been proven to be an effective MTF calibration method for a number of interferometric microscopes and a scatterometer [Nucl. Instr. and Meth. A 616, 172-82 (2010]. Here we report on a significant expansion of the application range of the method. We describe the MTF calibration of a 6 inch phase shifting Fizeau interferometer. Beyond providing a direct measurement of the interferometer's MTF, tests with a BPR array surface have revealed an asymmetry in the instrument's data processing algorithm that fundamentally limits its bandwidth. Moreover, the tests have illustrated the effects of the instrument's detrending and filtering procedures on power spectral density measurements. The details of the development of a BPR test sample suitable for calibration of scanning and transmission electron microscopes are also presented. Such a test sample is realized as a multilayer structure with the layer thicknesses of two materials corresponding to BPR sequence. The investigations confirm the universal character of the method that makes it applicable to a large variety of metrology instrumentation with spatial wavelength bandwidths from a few nanometers to hundreds of millimeters.
\end{abstract}

Keywords: surface metrology, surface profilometer, interferometric microscope, modulation transfer function, power spectral density, calibration, error reduction, fabrication tolerances, metrology of x-ray optics

\section{INTRODUCTION}

The modulation transfer function (MTF) is widely used to describe the spatial response of imaging devices and surface profilometers. ${ }^{1}$ The MTF of a profilometer accounts for the effects of the instrument's optical system, detector, signal processing, software algorithm, and environmental factors to the measured power spectral density (PSD) distributions of the surface height and slope (see e.g., Refs. ${ }^{2-6}$ and references therein). In the course of PSD measurements of a surface under test (SUT), the measured surface PSD, $P S D_{\text {measured }}$, is obtained by the square modulus of a straightforward discrete Fourier transform of the measured height distribution. To the extent that the response of the instrument can be characterized as a linear system, the measured PSD is a product of the PSD inherent for the SUT, PSD ${ }_{\text {SUT }}$, and the MTFs of the individual components (objective, detector, etc.) of the instrument:

$$
P S D_{\text {measured }}=P S D_{S U T} \times M T F^{2} .
$$

The MTF in Eq. (1) is the total MTF of the instrument. It can be experimentally determined by comparing the measured PSD distribution of a test surface to the corresponding ideal PSD distribution which is numerically simulated or found from PSD measurements with an instrument with significantly higher resolution. The square root of the ratio of the measured PSD distribution to the ideal PSD distribution gives the MTF of the instrument.

*vvyashchuk@lbl.gov; phone 1510 495-2592; fax 1510 486-7696; www-esg.lbl.gov/Production/OML/index.html 
A number of methods for MTF measurement have been developed. ${ }^{7-19}$ The effectiveness of a given method critically hinges on the appropriate choice of test surface. A successful test surface should be suitable for calibration over the entire instrumental field of view with a uniform sensitivity to the MTF over the entire spatial frequency range up to the Nyquist frequency of the instrument. Additionally, in order to be used as a certified standard, the MTF test surface should satisfy the conditions of ease of specification, reproducibility, and repeatability; and the accuracy of the MTF calibration should have a reasonably low sensitivity to possible fabrication imperfections of the surface. Most of the common test patterns used in MTF measurements, including knife-edge sources (step height standards), ${ }^{7-11}$ bar targets, ${ }^{12}$ sinusoidal surfaces, ${ }^{13}$ periodic and quasiperiodic patterns, ${ }^{14-16}$ white noise patterns ${ }^{17}$ and random reference specimens ${ }^{15,18}$ fail to meet all of these requirements. For a comprehensive review of standard reference specimens, see Ref. ${ }^{19}$ and references therein.

Recently, an original technique for precise measurement of the one- (1D) and two-dimensional (2D) MTF has been developed. The technique is based on the use of binary pseudo-random gratings (BPRG) and arrays. ${ }^{20,21}$ Unlike most conventional test surfaces, the inherent PSD of the BPR gratings and arrays has a deterministic white-noise-like character. This allows the direct determination of the one- (1D) and two-dimensional (2D) MTF, respectively, with a sensitivity uniform over the entire spatial frequency range of a profiler. The success of applying this method to different interferometric microscopes and a scatterometer has been experimentally demonstrated..$^{20-24}$

In the present work, we present results of extending the BPRA method to large field-of-view interferometers, a class of instruments that are, and will continue to be, a standard for making high precision surface height measurements over relatively low spatial frequency ranges from approximately $10^{-2} \mathrm{~mm}^{-1}$ to $10 \mathrm{~mm}^{-1}$. An extension of the method to the micro- and nano- scale measurements with scanning and transmission electron microscopes (SEM and TEM, respectively) is also presented. We provide details of the development of BPR test samples suitable for calibration of electron microscopes. These samples, which are multilayer structures with layer thicknesses corresponding to a BPR sequence, are fabricated with a focused ion beam technique. In the next section, we briefly overview the mathematical fundamentals of BPR sequences and arrays that are important for the MTF measurements. Section 3 discusses the development of the first prototype of the BPR test surfaces suitable for the MTF measurements with large aperture interferometers. Specifically, we present the results of MTF tests with a ZygoTM-GPI 6-inch Fizeau interferometer. The details of fabrication and use of a BPR multilayer structure, suitable for characterization of SEMs and TEMs, are presented in Sec. 4. The paper concludes (Sec. 5) by summarizing the main concepts discussed throughout the paper and stating a plan for extending the technique to calibrate other types of surface profilometers, including scanning probe microscopes. The investigations confirm the universal character of the method that makes it applicable to a large variety of metrology instrumentation with spatial wavelength bandwidths from a few nanometers to hundreds of millimeters.

\section{BPR SEQUENCES AND ARRAYS}

BPR sequences and arrays are one- and two- dimensional patterns, respectively, of statistically independent and uniformly distributed binary elements (1's and 0 's or -1 's and +1 's). We use the term 'pseudo-random' to emphasize that the distributions are generated by mathematically precise rules to be random in the mathematically strong sense. ${ }^{25-27}$ Alternatively, such sequences are referred to as pseudo-noise sequences or m-sequences. ${ }^{25}$

Particular methods for generation of pseudo-random sequences ${ }^{28,29}$ were developed in connection with communication and encryption processes, ${ }^{30}$ acoustics ${ }^{31}$, and pseudo-random chopping of a beam in time-of-flight experiments with slow neutrons ${ }^{32-37}$ and molecular beams. ${ }^{38-40}$ The maximum duty cycle (relative number of 1's and 0's) of approximately $50 \%$ is obtained with a maximum-length pseudo-random sequence (MLPRS). ${ }^{29,38,39}$ A sequence $\left\{a_{i}\right\}$ of $N$ elements ( $i=0,1, \cdots, N-1, N=2^{n}-1$, where $n$ is an integer) is qualified as a MLPRS, if (i) the autocorrelation of the sequence sums to $2^{n-1}$ and (ii) the sequence is 'almost' uncorrelated. The conditions (i) and (ii) are very natural for a purely random sequence, or white noise that consists entirely of uncorrelated binary elements with a $\delta$-function-like correlation function. According to the Wiener-Khinchin theorem, ${ }^{41}$ the PSD of a sequence with a $\delta$-function-like correlation function is a frequency independent white-noise-like distribution. The 1D PSD sequences used in this publication were generated using the algorithm described in detail in Ref. ${ }^{29}$

Two-dimensional analogues to the 1D BPR sequences are designated as uniformly redundant arrays (URA). URAs are widely used as optimal mask patterns for coded aperture imaging techniques. ${ }^{26}$ Analogous to the time-of-flight technique 
based on 1D BPR sequence chopping, ${ }^{32-40}$ an imaging technique based on the 2D URAs allows one to obtain a better signal-to-noise ratio, keeping the high angular resolution characteristic of a single pinhole aperture. ${ }^{42-44}$ Similar to the 1D BPR sequences, the URAs possess both high throughput (50\%) and a delta-function-like cyclical autocorrelation function that corresponds to a flat 2D PSD spectrum. Due to the similarity, we employ the term BPR array rather than URA, when discussing the 2D test surfaces used for the MTF calibration. The URA used in this paper follows the original prescription of the twin-prime class given in Ref. ${ }^{42}$ The URA is configured as a rectangular aperture of dimensions $r \times s$, where $r$ and $s$ are prime numbers and $r=s+2$. For more details on the URA generation algorithm see Refs. ${ }^{42,43}$

1D and 2D BPR gratings and arrays which we use for MTF calibration of surface profilometers (such as interferometric microscopes, ${ }^{21-24}$ scatterometers, ${ }^{23}$ and, now, large area interferometers) are sets of rectangular grooves pseudo-randomly distributed over a uniform 1D and 2D grid, respectively. Figure 1a illustrates the design of a BPR array. The pitch of the grid (width of the smallest element of the pattern), $\Delta$, determines the inherent Nyquist frequency of the BPR pattern, $f_{H}=1 / 2 \Delta$. For the lower spatial frequencies, the inherent PSD is limited by the whole size of the array, $r \Delta$ and $s \Delta$ for the two orthogonal directions, $f_{L x}=1 / r \Delta$ and $f_{L y}=1 / s \Delta$, respectively. The height of the pattern, $h_{0}$, determines the amplitude of the array's inherent PSD spectrum. Because the PSD from a BPR grating or array is a result of the groove distribution, it is not particularly sensitive to the groove shape or roughness of the groove surfaces, top or bottom. ${ }^{24}$ The only requirement is that the value of $h_{0}$ should be large enough to ensure a negligible contribution from fabrication imperfections to the PSD measurements.

Figure 1b illustrates a design of a BPR multilayer (ML) test sample suitable for measurements with electron microscopes. A BPRML sample is a multilayer structure consisting of two materials (marked with indexes 0 and 1 ) with significantly different contrasts when observed with an electron microscope. In order to serve as a test sample for MTF calibration of a SEM or a TEM, the layers of the two materials are pseudo-randomly distributed according to a binary pseudo-random sequence, similar to ones used for fabrication of BPR gratings and applied to 1D MTF calibration of interferometric microscopes. ${ }^{21,22}$ In the BPRML case, the thickness of a particular layer of the material with index 0 (1) is equal to the elementary thickness of the multilayer, $\Delta t$, multiplied by the number of adjacent 0 's ( 1 's) in the BPR sequence used for the multilayer generation.
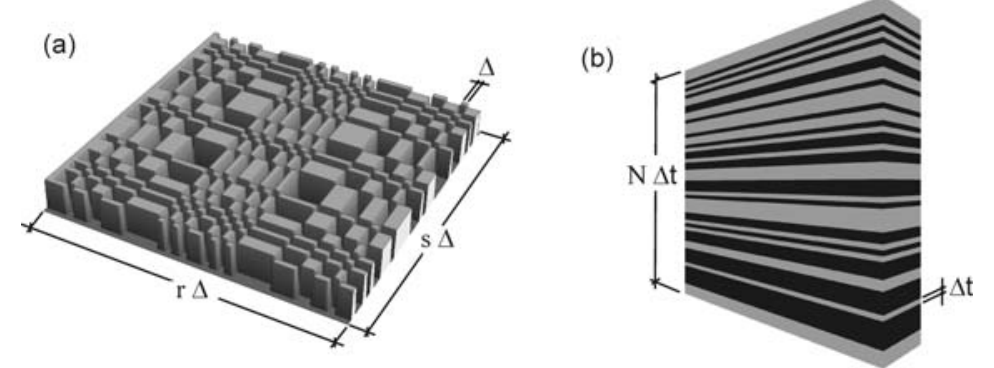

Figure 1. Examples of BPR test patterns: (a) 2D BPR array of $43 \times 41$ elements; (b) BPR multilayer consisting of 63 elementary sub-layers of thickness $\Delta t$. In order to generate the BPR patterns, the algorithms described in Refs. ${ }^{29,42}$ were used.

The BPR array and grating patterns used throughout the present work are generated with a maximum filling factor of about 50\% providing improved signal-to-noise ratio of the PSD spectra of the test surface. The inherent PSD spectrum of such a pattern is independent of spatial frequency (white-noise-like). Therefore, any deviation of a PSD spectrum measured with a real instrument from a white-noise-like spectrum is a measure of the instrumental MTF.

\section{BPR ARRAY SAMPLE FOR USE WITH LARGE APERTURE INTERFEROMETERS}

\subsection{BPRA fabrication}

Due to the deterministic binary character, BPR test surfaces are easy to specify for standard micro- and nano-fabrication processes. For the purpose of MTF measurement, an ideal surface based on a BPR pattern is determined as a set of rectangular grooves of binary height levels with grooves and peaks corresponding to values of 1 and 0 in the BPR 
sequence or array (Fig. 1). The optimal height, $h_{0}$, and fundamental element size (pitch), $\Delta$, depend on the specifics of the instrument under calibration. ${ }^{23}$

In order to carry out MTF calibration of an interferometer, an optimal BPRA sample should have a height step smaller than the light wavelength, $h_{0}<\lambda$, and fill a relatively large area of the instrumental field of view. In the case of the $\mathrm{Zygo}^{\mathrm{TM}}$-GPI interferometer this leads to a relatively stringent requirement that the figure error of the BPRA substrate be less than $\lambda / 20$ over a clear aperture of about $150 \mathrm{~mm}$. In addition to the high price of such substrates, they are also rather thick, $3 / 4$ in. -1 in., making it difficult to use them in conventional micro-lithography machines and processes. For this reason, the first prototype BPRA samples (with $4021 \times 4019$ elements) for MTF calibration of the Zygo ${ }^{\mathrm{TM}}$-GPI interferometer were fabricated by conventional micro-lithography with chromium deposition on a standard 4 in. silicon wafer with a thickness of $4 \mathrm{~mm}$.

Figure 2a shows the higher spatial frequency topography of the fabricated BPRA as it was seen with the MicroMap $^{\mathrm{TM}}-570$ interferometric microscope equipped with a $10 \times$ objective. The resolution of the microscope is adequate for examination of the quality of the profile of the BPRA elements. Based on the consideration of the effect of the BPRA fabrication imperfections on the MTF correction given in Ref., ${ }^{18}$ we conclude that the observed non-utility of the profile of the array elements is negligible, perturbing the inherent BPRA PSD distribution at higher spatial frequencies by less than $1 \%$. The BPRA's geometrical parameters are measured to be $h_{0} \approx 100 \mathrm{~nm}$ and $\Delta \approx 20 \mu \mathrm{m}$.
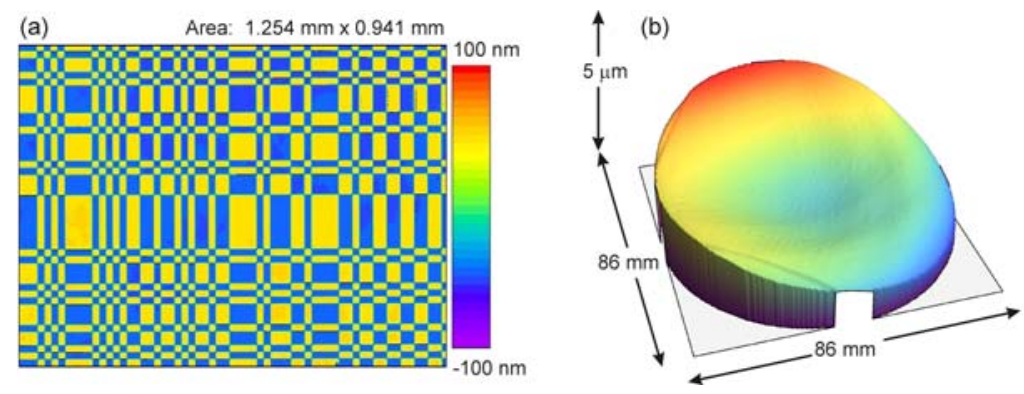

Figure 2. (a) Measurement of the BPRA height distribution with the Micromap ${ }^{\mathrm{TM}}-570$ interferometric microscope equipped with a $10 \times$ objective. (b) Measurement of the BPRA height distribution with Zygo ${ }^{\mathrm{TM}}$-GPI with the $1 \times$ magnification. The BPR surface structure is almost invisible due to the figure variation of the sample surface.

Unfortunately, the residual curvature and low frequency variations of the wafer used for fabrication of the BPRA were found to be much worse than that which is required for an ideal substrate, $<<h_{0}$. Measurements with the Zygo ${ }^{\mathrm{TM}}$-GPI with $1 \times$ magnification (Fig. 2b) revealed that the fabricated BPRA test surface has a significant curvature on the order of 1000 meters, which masks the appearance of the BPRA features, even at a larger magnification of the interferometer. Indeed, the measured peak-to-value variation of $\sim 5 \mu \mathrm{m}$ is much larger than the BPRA height $h_{0}=100 \mathrm{~nm}$.

A similar problem was encountered during the calibration of the MicroMap ${ }^{\mathrm{TM}}-570$ interferometric microscope when using the smaller magnifications, e.g. $2.5 \times$ and $5 \times$ objectives. ${ }^{22,24}$ In these cases waviness of the substrate tended to distort the measured PSDs from the PSDs expected to result from the mathematical properties of the BPRA. The solution to this problem was to etch the BPRAs into a super polished silicon substrate. Because the largest field of view with the MicroMap $^{\mathrm{TM}}-570$ microscope is only $2.51 \mathrm{~mm}$ by $1.88 \mathrm{~mm}$, a suitable substrate is relatively inexpensive and the corresponding developments are currently in progress at the Advanced Light Source optical metrology laboratory (OML).

In the case of the Zygo ${ }^{\mathrm{TM}}$-GPI, two mutually supplemental alternatives to starting with an excessively expensive substrate have been employed. Firstly, the overall lower spatial frequency variations of the substrate could be accurately fitted and subtracted using Zernike polynomials or filtered out with the Zygo software's data filtering options. An ideal fitting/filtering procedure would remove all low frequency irregularities without disturbing the higher spatial frequency structure of the measured PSD spectra. Secondly, the range of substrate figure variations can be significantly decreased if the MTF calibration is applied to the Zygo ${ }^{\mathrm{TM}}$-GPI interferometer with the maximum magnification (nominally $6 \times$ ). Such proof-of principle MTF measurements are the focus of the present work (see the next subsection). 


\subsection{MTF measurements with Zygo $^{\text {TM }}$-GPI interferometer}

The BPRA test sample was mounted in the front of the $\mathrm{Zygo}^{\mathrm{TM}}$-GPI interferometer on a rotatable kinematic mount and the square elements were visually aligned to the grid of the CCD. The small value of the fundamental period of the BPRA $(20 \mu \mathrm{m})$ allows for the measurement of the MTF of the Zygo ${ }^{\mathrm{TM}}$-GPI with maximum magnification, i.e. when the nominal pixel size of the CCD detector is about $90 \mu \mathrm{m}$. However, even at the maximum magnification, the BPRA surface figure measured over the whole field of view $(640 \times 480$ pixels) still exhibits significant low frequency variations. Neither detrending with a generalized quartic polynomial nor using a fast Fourier transform (FFT) filter with a low frequency cutoff, available in the Zygo software, fully removed these variations.

Nevertheless, Zygo MTF tests were still possible using a field of view of approximately $215 \times 215$ CCD pixels and measuring a central portion of the test surface, which had the least amount of curvature. The data were then filtered using a FFT filter with three different low frequency cutoffs, from $0.025 \mathrm{~mm}^{-1}$ to $0.25 \mathrm{~mm}^{-1}$ to $0.5 \mathrm{~mm}^{-1}$. Note that when filtering of this type is used, the dimensions of the data set are necessarily reduced. Figure 3 depicts 2D height distributions to visualize the effects of filtering the data compared with detrending using a generalized polynomial. Detrending removes a significant amount of the substrate's saddle like shape, but filtering clearly provides a better means for removing other low frequency variations.

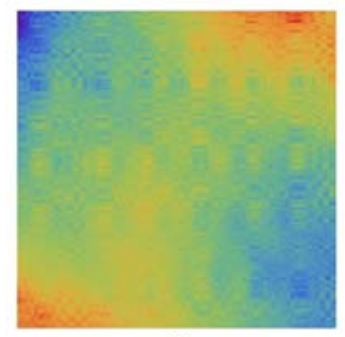

(1)

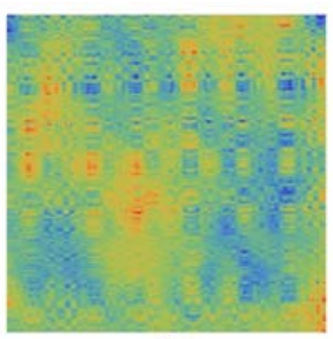

(2)

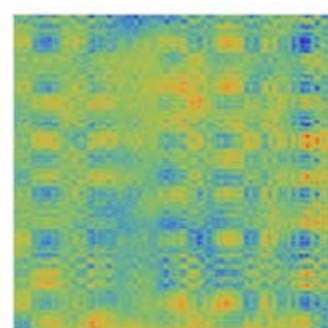

(3)

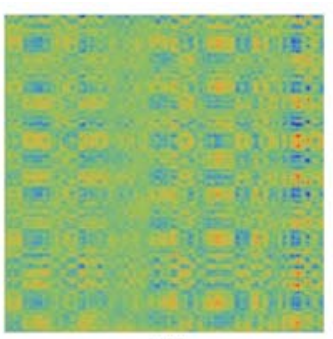

(4)

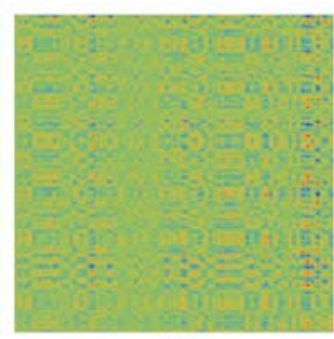

(5)

Figure 3. Two-dimensional height distribution plots of the BPRA measured with Zygo 6-inch Fizeau interferometer equipped with the nominal $6 \times$ magnification. (1) No filtering or detrending applied; (2) detrended using generalized quartic polynomial; (3), (4) and (5) data filtered using an FFT filter with low frequency cutoffs of $0.025 \mathrm{~mm}^{-1}$ to $0.25 \mathrm{~mm}^{-1}$ to 0.5 $\mathrm{mm}^{-1}$, respectively.

A comparison of the PSDs from the unprocessed data, the detrended data and the filtered data indicates positive results, Fig. 4. Both the tangential and sagittal PSDs before detrending or filtering demonstrate an inverse-power-like (a negative slope on a log-log scale) character. Detrending the data significantly reduces the PSD level at the lower spatial frequencies, but there is still a significantly raised lower frequency tail. As filtering is applied with increasing cutoff frequencies, however, the raised low frequency tail starts to flatten out and the PSDs start to exhibit precisely the expected characteristics for a BPRA test surface. That is, the PSD is largely flat across the lower frequency range before it begins to roll off in the higher frequency range, which is primarily an effect of the instrumental MTF. Note that the low frequency filtering does not cause any noticeable perturbations to the PSD at the higher frequency range (limited by the roll-off at the Nyquist frequency), interesting from the point of view of MTF measurement. Thus, FFT filtering is a suitable method for removing inherent waviness of the substrate and to recover the desired BPRA height distribution which can then be used for MTF calibration.

Another interesting result from the Zygo ${ }^{\mathrm{TM}}$-GPI MTF measurements is that the instrument's Nyquist frequencies, easily identified by visual inspection of the 1D PSDs, in the tangential and sagittal directions are significantly different. To determine the source of this asymmetry, the BPRA test surface was rotated ninety degrees from its original orientation and re-measured. Comparison of the measurements with and without rotation has confirmed that the asymmetry is inherent to the Zygo MetroPro Version 7.6.1 software that processes the data from rows and columns of the CCD in different ways. Note that a similar asymmetry of the tangential and sagittal PSD spectra has also been observed with another phase measuring Fizeau-type interferometer. ${ }^{44}$

The observed asymmetry is remarkably analogous to a discovery regarding how the MicroMap ${ }^{\mathrm{TM}}-570$ processes data. ${ }^{4,6}$ In the case of the MicroMap ${ }^{\mathrm{TM}}-570$, a detailed investigation of the origin of the anisotropy problem has been performed. ${ }^{4}$ The problem appears when a two field interlaced camera is used. ${ }^{45}$ One field consists of odd pixel lines, another includes the even lines. Both fields are collected simultaneously but are read out alternately. Such a read-out process leads to a systematic distortion between alternate detector outputs, corresponding to two fields. This distortion is reduced by 
averaging (summing) alternate outputs with the instrumental software. The averaging eliminates the distortion but also reduces the image resolution (Nyquist frequency) in the sagittal direction. Note that the latest (fifth) version of the MicroMap software accounts for the asymmetry by applying an additional averaging over two neighboring columns.
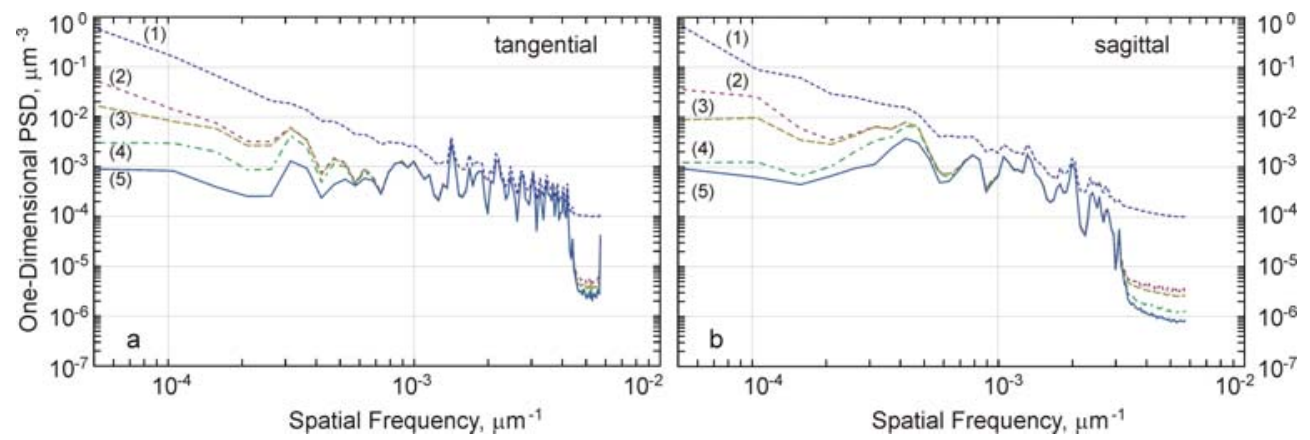

Figure 4. One-dimensional PSDs obtained from BPRA test sample height distribution measured with Zygo 6-inch Fizeau interferometer equipped with the nominal $6 \times$ magnification. (1) No filtering or detrending applied; (2) detrended using generalized quartic polynomial; (3), (4) and (5) data filtered using an FFT filter with low frequency cutoffs of $0.025 \mathrm{~mm}^{-1}$ to $0.25 \mathrm{~mm}^{-1}$ to $0.5 \mathrm{~mm}^{-1}$, respectively.

\section{BPR MULTILAYER TEST SAMPLES FOR USE WITH ELECTRON MICROSCOPES}

\subsection{Fabrication of BPR multilayer test samples}

Here we describe the details of the development of BPRML test samples suitable for measurements with scanning and transmission electron microscopes. First, we create a multilayer structure consisting of two materials, $\mathrm{WSi}_{2}$ and $\mathrm{Si}$ (marked with indexes 0 and 1 , respectively) with significantly different contrasts when observed with an electron microscope. In order to serve as a test sample for MTF calibration of a SEM or a TEM, the 1010 layers of the two materials have thicknesses pseudo-randomly distributed according to a binary pseudo-random sequence of 2047 total elements. The thickness of a particular layer of the material with index 0 (1) is equal to the elementary thickness of the multilayer, $\Delta t=3 \mathrm{~nm}$, multiplied by the number of adjacent 0 's (1's) in the BPR sequence used for the multilayer generation. The thickest deposited layer in the stack is $33 \mathrm{~nm}$, which corresponds to eleven of the same (0's or 1's) adjacent elements. The specified total thickness of the multilayer deposited is $6141 \mathrm{~nm}$. The first layer of $33 \mathrm{~nm}$ of $\mathrm{WSi}_{2}$ is on the substrate, while the top layer of $6 \mathrm{~nm}$ of $\mathrm{Si}$ is exposed to air. The overall size of the sample multilayer deposited on $0.5 \mathrm{~mm}$ thick $\mathrm{Si}(100)$ substrate is approximately $25 \mathrm{~mm} \times 12.5 \mathrm{~mm}$.

The BPR sequence of 2047 elements was generated using parameters ${ }^{29} n=11$ and $M=83$. Unfortunately, the recursion coefficient $M=83$ was used mistakenly (instead of $M=43$ ); it does not correspond to a recursion coefficient which produces an ideal MLPRS. Although the fabricated BPRML samples do not correspond to an ideal BPR distribution, the samples were found to be still suitable for MTF measurements with an SEM. As it was verified analytically, the autocorrelation function of the sequence is very close to a one-element delta-function expected for the corresponding BPR sequence (e.g., with $M=43$ ); and the inherent PSD spectrum is a white noise one, however, with a noticeable level of noise. A new BPR multilayer with the ideal BPR distribution of layers and with even smaller elementary thickness $(1.5 \mathrm{~nm})$ and larger total number of elementary layers (4095) is in progress.

The BPR multilayer was fabricated with solid-source targets of B-doped Si and hot-pressed $\mathrm{WSi}_{2}$ using modified 3-inch direct-gas injection cathodes ${ }^{46}$ in a turbo-pumped rotary deposition system. The target to sample distance was $78 \mathrm{~mm}$, with a process gas pressure of Ar held at a constant 2.3 milliTorr by upstream dual-MFC feedback control. The average gas flow through each cathode was $~ 9$ SCCM. Deposition was carried out at a constant power of 170 watts for both guns. The proper thickness for each individual layer is produced by raster-scanning the substrate over figured apertures by varying both the number of passes over this aperture and the rotational velocity. Due to the inherent nature of magnetron deposition growth rate to decay over time, a compensation factor is included during the growth which adjusts the velocity appropriately as is used for growth of other types of thick multilayers. ${ }^{47,48}$

Test sample preparation and the SEM and TEM measurements with the samples were performed at Evans Analytical Group, Inc. ${ }^{49}$ The BPRMLs were loaded in and processed with a Dual Beam FIB (focused ion beam)/SEM instrument 
(Helios NanoLab, FEI Company ${ }^{\mathrm{TM}}$ ). The instrument integrates imaging capabilities of a field emission SEM and the capability for preparation of a precise thin sample cross section using a focused ion beam. In order to avoid rounding of the top surface edge of the sample cross section in the course of FIB etching, the area of the BPRML used for the SEM measurements was preliminary coated with a thin, about $1.5 \mu \mathrm{m}$ thick, layer of Pt.

For the SEM measurements, the BPRML was cross-sectioned by etching with the FIB/SEM technique - Fig. 5a. After careful FIB flattening of the side wall of the dimple shown in Fig. 5a, the dissected BPR multilayer cross-section was measured with a SEM - Sec. 4b.

The process for fabrication of a test sample suitable for measurements with a TEM consisted in FIB etching out of a thin sample from the BPRML - Fig. 5b. The TEM sample preparation was performed with the multilayer piece shown in Fig. 5b when it was completely detached from the multilayer. In this step, in order to hold the piece, a sharp transporting needle was Pt ion-beam welded to the free, right-hand side of the piece. After that, the piece was cut out from the rest of the BPRML and attached to a pin of a standard TEM sample holder. Finally, in order to decrease the test sample piece thickness to 60-100 nm and make the thickness uniform, both sides of the ML piece at its free end were processed with 'super-polishing' at extremely low FIB current.
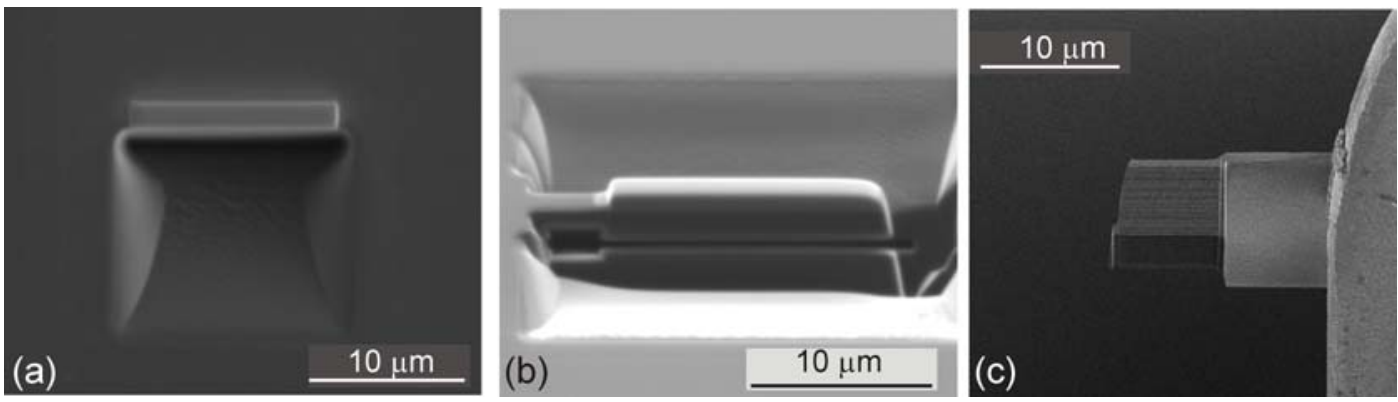

Figure 5. (a) Photomicrograph of the BPRML with a dimple etched with the FIB. In the course of FIB etching, deposition of the etched material leads to overgrowing of the sides of the dimple. The sample was used for the SEM measurements discussed in Sec. 4.2. (b) Photomicrograph of the BPRML with sample multilayer section detached from the Si substrate by FIB etching and undercutting across the Si substrate cross-section. The sample was used for the TEM measurements discussed in Sec. 4.3. (c) Photomicrograph of the multilayer test sample piece under 'super-polishing' to approximate $80 \mathrm{~nm}$ thickness suitable for TEM imagining.

Figure 5c shows an SEM image of the BPRML TEM sample prepared using the FIB/SEM process described above. Because SEM imaging is associated with a noticeable ablation of the sample material, the last SEM measurement was carried out just before the last cycle of the FIB 'super-polishing.' After the FIB/SEM preparation of the BPRML TEM sample was completed, the FIB/SEM vacuum chamber was vented and the holder with the sample was moved to a clean glass dish. In this way the sample was brought to a TEM lab.

\subsection{SEM measurements of the BPRML cross-section.}

SEM measurements of the multilayer cross section (Fig. 5a) prepared with the FIB technique were performed with an electron beam tilted by $52^{\circ}$ with respect to the surface normal. This led to a distortion of the vertical scale of the SEM photomicrograph of the cross sections of the samples. The distortion should be accounted for when estimating the thickness of the layers and calculating the PSD spectra. Figure 6a shows an SEM image of a cross section of the BPRML sample obtained with $35,000 \times$ magnification. The vertical bar in the image is placed in order to provide a corrected vertical scale that accounts for the $52^{\circ}$ tilt of the electron beam.

Magnification of $35,000 \times$ is suitable for acquiring an image of the entire ML cross-section; however, it is too low to resolve the thinnest layers of the BPRML structure. SEM images of the BPRML samples measured with a significantly increased magnification $(200,000 \times)$ are shown in Fig. 6b. It still seems that magnification of $200,000 \times$ is not enough to resolve the thinnest layers of the BPRML structure. Images with significantly higher resolution were obtained using FIB/TEM technology (Sec. 4.3).

As an example of the valuable information that can be obtained with the developed BPRML test samples, in Fig. 6c we show how the measurements of the BPRML lead to the understanding of the limitations of the FIB/SEM analysis. Figure 6c presents the 1D PSDs calculated for the "top third," (dashed line), "middle third" (dash-dotted line), and "bottom 
third” (solid line) of the BPRML image obtained with 35,000× magnification (Fig. 6a). The observed difference of the spectra suggests that the SEM images performed at a tilt angle of $52^{\circ}$ suffer from a limited focal depth of the instrument. As a result, for a vertically lengthy sample, such as our BPRML cross-section, the image resolution varies significantly in vertical direction.
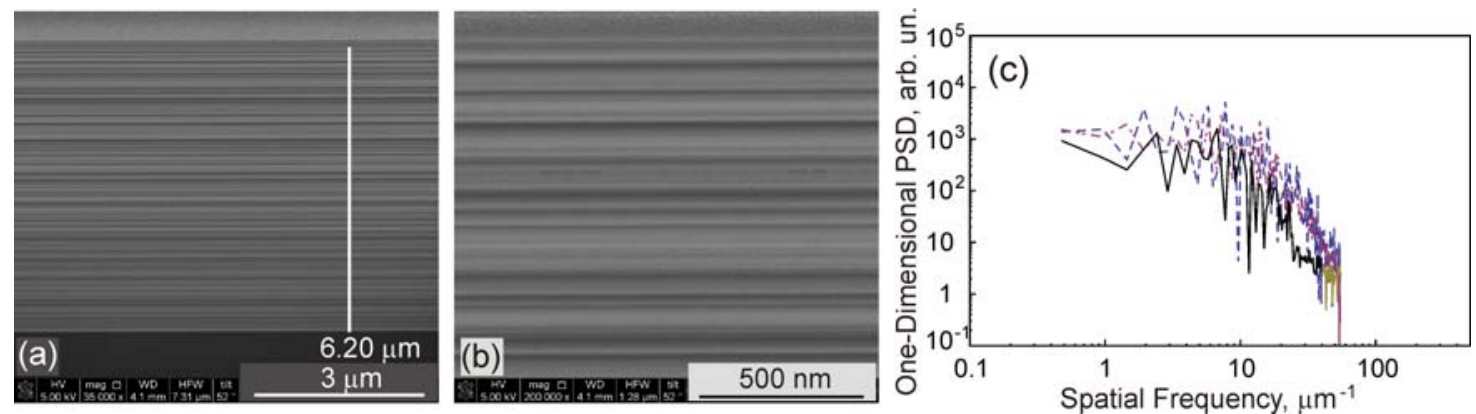

Figure 6a: (a) SEM image of a cross section of the BPRML (see Fig. 5a) obtained with 35,000× magnification. (b) SEM image of a cross section of the BPRML obtained with 200,000× magnification. (c) Power spectral densities calculated for the "top third" (blue dashed line), "middle third" (red dash-dotted line), and "bottom third" (black solid line) of the image shown in (a). The defocusing effect is clearly seen as a lower spatial frequency shift of the PSD spectra for the bottom third part of the image.

In order to obtain the PSD distributions from the SEM measurements, the images in tiff-format (as ones shown in Fig. 6) were analyzed by converting the image brightness profiles to 1D PSD distributions. We used the following PSD processing. First, in order to avoid a contribution to the PSD spectrum from a gate-like function associated with the averaged image brightness, an original 2D brightness distribution was detrended by subtracting the best fitted plane surface. As a result, the averaged brightness of the detrended image is equal to zero (a negative brightness value is now allowed). Second, after detrending, the 1D PSD distributions were calculated for each column and averaged to decrease random spectral variation in the way described, e.g., in Refs. ${ }^{4-6}$ The PSD spectra at this stage have a characteristic high frequency roll-off that is due to the limited resolution of the SEM. The spectra flatten at significantly high frequencies where a contribution of the instrumental random noise exceeds the PSD magnitude inherent to the image of the structure. Finally, the random noise spectrum is removed by subtracting a minimum PSD value of the flat tail. In this way, we avoid obtaining a negative PSD value that is unphysical.

\subsection{TEM measurements with the BPRML sample prepared with SEM/FIB technique}

The structure of the BPRML TEM sample was investigated with a Tecnai ${ }^{\mathrm{TM}} \mathrm{TEM}$ instrument (FEI, Co.). The instrument is capable of high-resolution transmission electron and scanning transmission electron microscopy (STEM). With electron energies of about $300 \mathrm{keV}$ and with ultra-thin samples, the TEM image resolution is on the order of $1-2$ Angstroms. Compared to SEM, the TEM has better spatial resolution, and is capable of additional analytical measurements, but requires significantly more sample preparation, as was described above.

Figure 7 shows the TEM images of the BPRML sample piece (Fig. 5c) obtained with different magnifications. The resolution of the image, obtained at a rather low electron energy of $2.3 \mathrm{keV}$ (Fig. 7a), is noticeably higher than that of the image obtained with the SEM and shown in Fig. 6a. There is a noticeable contrast variation from top to bottom of the image that is due to the variation of the sample thickness. The contrast variation of a TEM image can be significantly improved by detrending the image with a low order polynomial 2D distribution. The specified relative accuracy of distance measurements with the SEM and TEM is $2-3 \%$. This probably explains the difference of the thickness values obtained with the instruments (compare the scale lines in Figs. 6a and 7a). Note that in the TEM images we did not see any noticeable imperfection of the multilayer structure. This is in contrast to a few of the measurements with the SEM in which we saw waviness in the layers.

The measurement in Fig. 7b was performed at an electron energy of $17.5 \mathrm{keV}$. The corresponding resolution is high enough to provide high contrast separation, even for the thinnest layers with $3 \mathrm{~nm}$ thickness. High resolution (at $255 \mathrm{keV}$ electron energy) TEM measurements of the BPRML test sample are shown in Figs. 7c and 7d. The TEM resolution is about $2 \AA$, allowing for the observation of the inter-layers of the ML coating. Moreover, in Fig. $7 \mathrm{c}$ an amorphous layer of Pt deposition is visible on the top of the sample, and a layer of oxidized Si is clearly seen on the top of the Si substrate (bottom of Fig. 7d). Note the very high contrast between the $\mathrm{WSi}_{2}$ and $\mathrm{Si}$ layers. The observed high stability of the 
measurements allows for effective and reliable stitching of multiple images taken along the sample cross-section. Processing of TEM measurements with stitching will be reported elsewhere. The major conclusion from the presented data is that the TEM measurements testify to the high quality of fabrication of the BPR multilayer with a structure exactly corresponding to the binary sequence that was used.
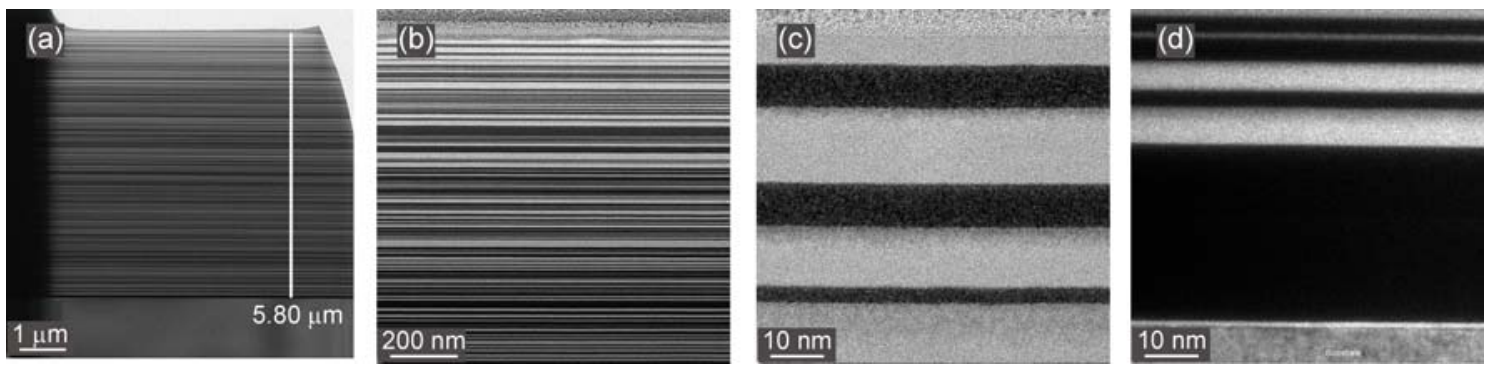

Figure 7a: (a) TEM images of a cross section of the BPRML sample piece obtained: at an electron energy of (a) $2.3 \mathrm{keV}$, (b) $17.5 \mathrm{keV}$, and (c,d) $255 \mathrm{keV}$. The contrast variation from top to bottom of the low magnification image (a) is due to the variation of the sample thickness. The images in (b) and (c) were taken over areas at the top of the BPRML test sample. The image in (d) approximately corresponds to the bottom part of the sample.

Similar to the PSD treatment of the SEM images applied in Sec. 4.2, the images in tiff-format obtained with the TEM (Figs. 7) were analyzed by converting the image brightness profiles to 1D PSD distributions. Figure 8a shows the resulting 1D PSD distribution along the vertical direction of the BPRML sample (Fig. 5c) obtained by averaging of six PSDs of the image files measured with $17.5 \mathrm{keV}$ electron energy, as shown in Fig. $7 \mathrm{~b}$.
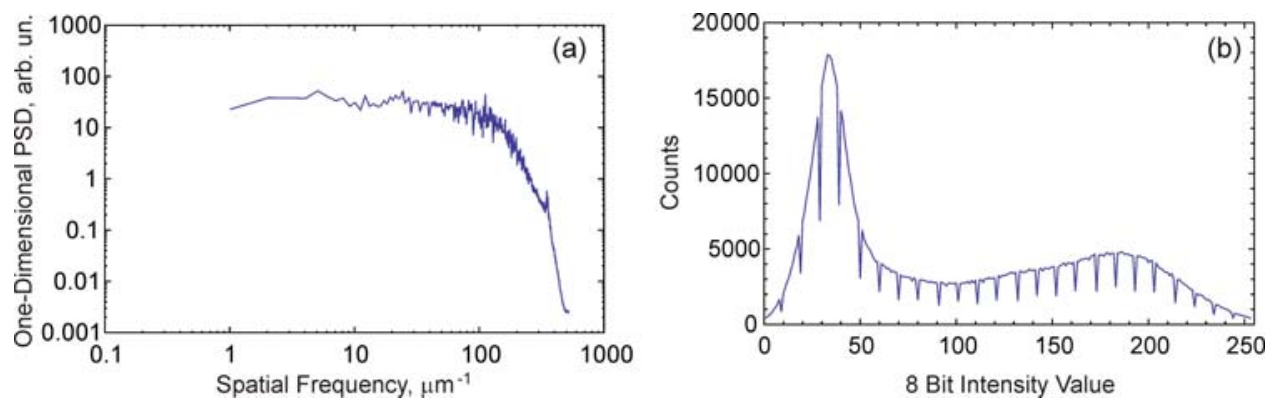

Figure 8. (a) 1D PSD of the BPRML sample obtained from the average of the PSDs obtained from 6 individual image files as measured with an electron energy of $17.5 \mathrm{keV}$ (Fig. 7b). (b) Histogram of intensity values obtained from a single TEM image measured with $17.5 \mathrm{keV}$ electron energy.

In the PSD distribution in Fig. 8a, there is a noticeable spike occurring at about $350 \mathrm{~mm}^{-1}$. This is most likely due to diffraction related to the nominal $3 \mathrm{~nm}$ fundamental layer thickness. In fact, after calibrating the length scale of the images, it was found that the fundamental layer thickness is about $2.8 \mathrm{~nm}$, which would exactly correspond to a diffraction peak at $350 \mu^{-1}$. A similar diffraction peak has been observed in scatterometer measurements with a BPR array, when the wavelength of the scattered light was smaller than a fundamental size of the array. ${ }^{23}$

The PSD measurements with the TEM suggest a number of questions related to a metrological interpretation of the data. One of the questions is about the high frequency behavior of the PSD spectra in Fig. 8a. Indeed, because the magnified pixel size is about $1 \mathrm{~nm}$, and the BPRML fundamental thickness is about $3 \mathrm{~nm}$, the TEM measurements are oversampled. In this case, one would expect the high frequency behavior of the PSDs to resemble a Sinc squared function. This is not the case for the spectra shown in Fig. 8a.

Another question is about the frequency distribution of different intensities in the TEM images recorded with 8-bit resolution. Figure $8 \mathrm{~b}$ show a histogram of intensity values obtained from a TEM image spread over $2^{8}$ intensity intervals. For the BPR sequence used here, the distribution of the BPRM contrasts (transmissions) should have two equal intensity frequency peaks corresponding to the low and high transmission materials. However, the histogram in Fig. 8b demonstrates a significant asymmetry in the appearance of the low and high intensities.

Figure 8b illustrates one more problem with TEM data presentation via tiff-files. The surprising (at first glance) spikes in the intensity histogram are probably related to the well known effect of double compression in the TIFF (MPEG) format. 
Therefore, in order to use TEM data for a reliable metrological characterization of a sample under test, one should first address the listed problems. An investigation of these problems and the appropriate way to handle them is currently in progress.

\section{CONCLUSION}

Suitable BPR test samples have been fabricated and used for MTF calibration and characterization of large aperture Fizeau interferometers and scanning and transmission electron microscopes.

The described MTF measurements with the $\mathrm{Zygo}^{\mathrm{TM}}$-GPI have revealed an asymmetry in the measurement along the tangential and sagittal directions. The next step is to fabricate a BPRA test standard on a substrate with high surface flatness. A relevant analytic model suitable for parameterization of the MTF of an interferometer based on measurements with the BPRA standard should also be developed.

The preceding SEM measurements with the developed BPR multilayer structure have shown that the SEM images performed at a tilt angle of $52^{\circ}$ suffer from a limited focus depth of the instrument.

The TEM measurements of the FIB/SEM developed BPRML test samples have confirmed the high quality of the BPR multilayer deposited at NSLS-II. The sharp image contrast between the WSi2 and Si layers has been demonstrated by super high resolution measurements with a scanning TEM. We have demonstrated that it is possible to get significant information about metrological reliability of the TEM measurements even for the case when the fundamental frequency of the BPRML sample is smaller by a factor of 1.5 than the Nyquist frequency of the measurements. The measurements bring out a number of problems related to the interpretation of the TEM data.

We plan to develop a BPRML sample consisting of about 4000 layers with an elementary thickness of $1.5 \mathrm{~nm}$. Such a sample would be more suitable for MTF characterization of electron microscopes with higher resolution. We are also working on building an analytical approximation of the measured PSD distributions as they relate to the theoretical PSD inherent to the samples, which would allow us to numerically evaluate the SEM resolution and account the effect of the defocusing.

In conclusion, the MTF calibration method using BPR test surfaces has a significantly universal applicability. It has already been adapted to a number of profiling instruments including interferometers, interferometric microscopes, scatterometers, scanning and transmission electron microscope. The existing nanofabrication methods and sample preparation methods, such as FIB sample preparation, are capable of fabricating BPRML test samples suitable for characterization of scanning probe microscopes. The corresponding developments and experiments are in progress at the Advanced Light Source Optical Metrology Laboratory in collaboration with the LBNL Center for X-Ray Optics and the NSLS-II/ BNL optics group.

\section{ACKNOWLEDGEMENTS}

The authors are grateful to David Susnitzky, Mark Izquierdo, and Udit Sharma for the FIB/SEM sample preparation and the TEM measurements. The Advanced Light Source is supported by the Director, Office of Science, Office of Basic Energy Sciences, Material Science Division, of the U.S. Department of Energy under Contract No. DE-AC0205CH11231 at Lawrence Berkeley National Laboratory. Research at Brookhaven National Laboratory is sponsored by the U.S. Department of Energy under Contract No. DE-AC02-98CH10886.

This document was prepared as an account of work sponsored by the United States Government. While this document is believed to contain correct information, neither the United States Government nor any agency thereof, nor The Regents of the University of California, nor any of their employees, makes any warranty, express or implied, or assumes any legal responsibility for the accuracy, completeness, or usefulness of any information, apparatus, product, or process disclosed, or represents that its use would not infringe privately owned rights. Reference herein to any specific commercial product, process, or service by its trade name, trademark, manufacturer, or otherwise, does not necessarily constitute or imply its endorsement, recommendation, or favoring by the United States Government or any agency thereof, or The Regents of the University of California. The views and opinions of authors expressed herein do not necessarily state or reflect those of the United States Government or any agency thereof or The Regents of the University of California. 


\section{REFERENCES}

[1] Boreman, G. D., [Modulation Transfer Function in Optical and Electro-optical Systems], SPIE Press, Bellingham, Washington (2001).

[2] Bracewell, R. N., [The Fourier Transform and Its Applications], McGraw-Hill Publishing Company, New York (1986).

[3] Elson, J. M., Bennett, J. M., "Calculation of the power spectral density from surface profile data," Appl. Opt. 34(1), 201-208 (2001).

[4] Yashchuk, V. V., Franck, A. D., Irick, S. C., Howells, M. R., MacDowell, A. A., McKinney, W. R., "Two dimensional power spectral density measurements of x-ray optics with the MicroMap interferometric microscope," Proc. SPIE 5858, 58580A-1-12 (2005).

[5] Yashchuk, V. V., Irick, S. C., Gullikson, E. M., Howells, M. R., MacDowell, A. A., McKinney, W. R., Salmassi, F., Warwick, T., "Cross-check of different techniques for two dimensional power spectral density measurements of $\mathrm{x}$ ray optics," Proc. SPIE 5921, 59210G-1-12 (2005).

[6] Yashchuk, V. V., Gullikson, E. M., Howells, M. R., Irick, S. C., MacDowell, A. A., McKinney, W. R., Salmassi, F., Warwick, T., Metz, J. P., Tonnessen, T. W., "Surface roughness of stainless-steel mirrors for focusing soft x-rays," Appl. Opt. 45(20), 4833-42 (2006).

[7] Barakat, R., "Determination of the optical transfer function directly from the edge spread function," JOSA 55(10), 1217-1219 (1965).

[8] Tatian, B., "Method for obtaining the transfer function from the edge response function," JOSA 55(8), 1014-1019 (1965).

[9] Creath, K., "Calibration of Numerical aperture effects in interferometric microscope objectives," Appl. Opt. 28(15), 3333-3338 (1989).

[10] Takacs, P. Z., Li, M. X., Furenlid, K., Church, E. L., "Step-height standard for surface-profiler calibration," Proc. SPIE 1995, 235-244 (1993).

[11] Harasaki, A. and Wyant, J. C., "Fringe modulation skewing effect in white-light vertical scanning interferometry," Appl. Opt. 39, 2101-2106 (2000).

[12] Boreman, G. D., and Yang, S., "Modulation transfer function measurement using three- and four-bar targets," Appl. Opt. 34, 8050-8052 (1995).

[13] Marchywka, M., Socker, D. G., "Modulation transfer function measurement technique for small pixel detectors," Appl. Opt. 31(34), 7198-7213 (1992).

[14] Nijhawan, O. P., Datta, P. K., Bhushan, J., "On the measurement of MTF using periodic patters of rectangular and triangular wave-forms," Nouv. Rev. Optique 6(1), 33-36 (1975).

[15] Rhee, H. G., Vorburger, T. V., Lee, J. W., and Fu, J., "Discrepancies between roughness measurements obtained with phase shifting interferometer and white-light interferometry," Appl. Opt. 44(28), 5919-5927 (2005).

[16] Chu, J., Wang, Q., Lehan, J. P., Gao, G., and Griesmann, U., "Measuring the phase transfer function of a phaseshifting interferometer," Proc. SPIE 7064, 7064C-1-8 (2008).

[17] Levy, E., Peles, D., Opher-Lipson, M., and Lipson, S. G., "Modulation transfer function of a lens measured with a random target method," Appl. Opt. 38(4), 679-683 (1999).

[18] Wolfe, C. R., Downie, J. D., and Lawson, J. K., "Measuring the spatial frequency transfer function of phasemeasuring interferometers for laser optics," Proc. SPIE 2870, 553-557 (1996).

[19] Song, J. F. and Vorbuerger, T., "Standard reference specimens in quality control of engineering surfaces," J. Res. of the Natl. Inst. Stand. Technol. 96, 271-289 (1991).

[20] Yashchuk, V. V., McKinney, W. R., and Takacs, P. Z., "Binary Pseudorandom Grating Standard for Calibration of Surface Profilometers," Opt. Eng. 47(7), 073602-1-5 (2008).

[21] Yashchuk, V. V., McKinney, W. R., and Takacs, P. Z., "Binary Pseudorandom Grating as a Standard Test Surface for measurement of Modulation Transfer Function of Interferometric Microscopes," Proc. SPIE 6704, 670408/1-12 (2007).

[22] Barber, S. K., Soldate, P., Anderson, E. D., Cambie, R., McKinney, W. R., Takacs, P. Z., Voronov, D. L., and Yashchuk, V. V., "Development of Pseudo-random Binary Gratings and Arrays for Calibration of Surface Profile Metrology Tools," J. Vac. Sci. and Tech. B 27(6), 3213-3219 (2009).

[23] Barber, S. K., Anderson, E. D., Cambie, R., McKinney, W. R., Takacs, P. Z., Stover, J. C., Voronov, D. L., and Yashchuk, V. V., "Binary Pseudo-Random Gratings and Arrays for Calibration of Modulation Transfer Function of Surface Profilometers," Nucl. Instr. and Meth. A 616, 172-182 (2010). 
[24] Barber, S. K., Soldate, P., Anderson, E. D., Cambie, R., Marchesini, S., McKinney, W. R., Takacs, P. Z., Voronov, D. L., Yashchuk, V. V., "Binary Pseudo-random Gratings and Arrays for Calibration of the Modulation Transfer Function of Surface Profilometers: Recent Developments," Proc. SPIE 7448, 744802-1-12 (2009).

[25] Bardell, P. H., McAnney, W. H., Savir, J., [Built-in test for VLSI pseudorandom techniques], John Wiley and Sons, Inc., New York (1987).

[26] Busboom, A., Elders-Boll, H., Schotten, H. D., "Uniformly Redundant Arrays," Experimental Astronomy 8, 97-123 (1998).

[27] Sclar, B., [Digital Communications: Fundamentals and Applications], $2^{\text {nd }}$ Ed., Prentice Hall (2001).

[28]Etzon, T., "Construction for perfect maps and pseudo-random arrays," IEEE Trans. on Information Theory 34(5), 1308-16 (1988).

[29] Koleske, D. D., and Sibener, S. J., "Generation of pseudo-random sequence for use in cross-correlation modulation, " Rev. Sci. Instrum. 63(8), 3852-3855 (1992).

[30] Mitra, A., "On Pseudo-Random and orthogonal Binary Spreading Sequences," Int. J. Information Techn. 4(2), 137144 (2008).

[31]Chu, W.T., "Impulse-Response and Reverberation-Decay Measurements Made by Using a Periodic Pseudorandom Sequence, " Appl. Acoustics 29, 193-205 (1990).

[32] Stern, T.E., Blaquiere, A., Valat, J., "Reactivity Measurement Using Pseudo-Random Source Excitation," J. Nucl. Energy Pt. A and B 16, 499 (1962).

[33] Gordon, J., Kroo, N, Orban, G., Pal, L., Pellionisz, P., Szlavik, F., Vizi, I., "Correlation Type Time-of-Flight Spectrometer with Magnetically Pulsed Polarized Neutrons," Phys. Lett. A 26, 122 (1968).

[34] Skold, K., "A Mechanical Correlation Chopper for Thermal Neutron Spectroscopy," Nucl. Instr. and Meth. 63, 114116 (1968).

[35] Virjo, A., "Statistical Analysis of Cross-Correlation Chopper for Time-of-Flight Measurements," Nucl. Instr. and Meth. 63, 351-352 (1968).

[36] Virjo, A., "The Fourier method in Slow neutron time-of-flight Spectrometry with a Pseudo-Random Input Signal," Nucl. Instr. and Meth. 73, 189-199 (1969).

[37] Virjo, A., "Slow Neutron Time-of-Flight Spectrometry with a Pseudo-Random Input Signal," Nucl. Instr. and Meth. 75(1), 77-84 (1969).

[38] Hirschy, V. L., and Aldridge, J. P., "A Cross Correlation Chopper for Molecular Beam Modulation," Rev. Sci. Instrum. 42(3), 381-383 (1971).

[39] Comsa, G., David, R., and Schumacher, B. J., "Magnetically suspended cross-correlation chopper in molecular beam-surface experiments," Rev. Sci. Instrum. 52(6), 789-791 (1981).

[40] Yashchuk, V. V., Ashkinadzi, B. N., Groshev, M. N., Ezhov, V. F., Isaev, T. A., Knyazkov, V. A., Krygin, G. B., Ryabov, V. L., "Cross-Correlation Time-of-Flight Spectrometer of Gas-Dynamic Molecular Beams," Instruments and Experimental Techniques 40(4), 501 (1997).

[41] Kay, S. M., [Modern Spectral Estimation: Theory and Application], Prentice Hall, Englewood Cliffs (1988).

[42] Fenimore, E. E. and Cannon, T. M., "Coded aperture imaging with uniformly redundant arrays," Appl. Opt. 17(3), 337-347 (1978).

[43] Caroli, E., Stephen, J. B., Cocco, G. Di, Natalucci, L., Spizzichino, A., "Coded aperture imaging in x- and gammaray astronomy," Space Science Reviews 45, 349-403 (1987).

[44] Gullikson, E. M., private communication.

[45] Holst, G. C., [CCD arrays, Cameras, and Displays], SPIE Optical Engineering Press, Bellingham (1998).

[46] Materials Science, Inc., http://www.msi-pse.com/Polaris.htm.

[47] R. Conley, C. Liu, J. Qian, C. M. Kewish, A. T. Macrander, H.Yan, H. C. Kang, J. Maser, and G. B. Stephenson, "Wedged multilayer Laue lens," Rev. Sci. Instrum. 79, 053104 (2008).

[48] R. Conley, C. Liu, C.M. Kewish, A.T. Macrander, C. Morawe, "Multilayer Growth in the APS Rotary Deposition System," Proc. SPIE 6704, 670505 (2007).

[49] Evans Analytical Group, Inc., http://www.eaglabs.com.

[50]Wang, W., and Farid, H., "Exposing Digital Forgeries in Video by Detecting Double MPEG Compression," MM\&Sec2006, Geneva, Switzerland (2006).

[51] Popescu, A., and Farid, H., "Statistical Tools for Digital Forensics," In 6th International Workshop on Information Hiding, Toronto, Canada (2004). 